\title{
Nanostructured Lipid Carriers (NLC): A Targeting Approach to the Brain via Intranasal Administration
}

\author{
Swatantra Kumar Singh Kushwaha*, Neelottama Kushwaha, Bushra Fatma, Piyush Pandey \\ Pranveer Singh Institute of Technology, Kanpur, Uttar Pradesh, INDIA.
}

\begin{abstract}
Different barriers like a blood-brain barrier in the brain restrict the transport of potential therapeutic elements for direct entry into the brain. For delivery of a wide range of therapeutic drugs directly to the brain can be achieved by direct targeting the brain via an olfactory and trigeminal neural pathway which bypasses the blood-brain barrier hence has gained more importance and considered as an accurate route of drug targeting to brain. Intranasal route transports the drug by delivering it directly to the brain and avoiding the systemic absorption which also avoids the side effect of enhancing the efficacy of nano therapeutics. As these types of drug delivery commonly targeted drug delivery to the brain via nose are complex. Different strategies applied for overcoming these challenges has been covered. Drugs to be transported through this system are usually carried out through nano particulate system known as nanotechnology which helps in transportation of drug particles directly to the central nervous system and participates in drug release through a carrier-mediated system called nano particulate
\end{abstract}

system have been extensively covered within the article. Parallel to this recent advancement in brain targeted drug delivery has been thoroughly explained and characterized. Although direct drug delivery to the brain is a vital challenge for researchers which can be overcome by using different types of strategies that have been covered under this article.

Key words: Nanostructured lipid carriers, Targeting, Intranasal, Brain, Delivery.

\section{Correspondence}

\section{Prof. Swatantra KS Kushwaha}

Associate Professor, Pranveer Singh Institute of Technology, Kanpur-209305, Uttar Pradesh, INDIA.

Phone no: +919450560321

Email: swatantrakushwaha15@gmail.com

DOI: 10.5330/ijpi.2020.3.46

\section{INTRODUCTION}

Based on recent scenario changes in lifestyle, work stress, irregular schedule and other factors such as pollution, environmental free radicals and higher exposure of toxic chemicals lead to several neurological disorders and disease. ${ }^{1,2}$ There are various neurological diseases, among that promptly growing case of dementia touched threatening level. It is estimated that the population suffering from this disease will be doubled in the next 20 years. ${ }^{3}$ Due to which it has drawn a lot of attentiveness and concern from the scientific community, where they have labeled this worldwide (25\% of cases from Europe followed by $49 \%$ and $18 \%$ from India and America). ${ }^{4}$

Neurodegenerative disorder (ND) is a term usually used for conditions that primarily affect the nervous system. Neurodegenerative disorder results in continuous damage to nerve cells which are the building block of the nervous system. The degradation may cause chronic, progressive loss of neurons which decreases the cognitive abilities of the brain such as the mental functioning of the brain (dementia) or movement problem (ataxia). It is a challenge to traditional drug delivery approaches for the effective delivery of drugs to the brain and central nervous system (CNS) because of the protective anatomy of the brain. Clinical potency of the therapeutic agent is not only depending on its bioavailability but also on its ability to penetrate the protective layer, i.e., blood-brain barrier (BBB) and cerebrospinal fluid (CSF).

There are several invasive techniques like intravenous, intrathecal, intraparenchymal and non-invasive techniques like chemical modification, prodrug approach, sublingual delivery and conjugation of drugs with ligands and antibody have been used to target the drug to the central nervous system (CNS). ${ }^{5}$ The olfactory route and peripheral circulation is a linkage between the brain and nose. The intranasal drug delivery route has achieved the interest of researchers as it's a potential delivery route for targeting the brain. ${ }^{6}$ Intranasal drug delivery is not a novel approach; it has been used traditionally for the administration of psychotherapeutic and another compound in the name of "Nasya Rasayana". During intranasal drug delivery, the drug molecule is absorbed through the nasal mucosa and reaches olfactory which eases the non-invasive entry of molecule into the brain to achieve the therapeutic effect. ${ }^{8}$

\section{DIFFERENT STRATEGIES ADOPTED FOR BRAIN TARGETING DRUG DELIVERY}

There are various types of drug delivery systems adopted for targeting drugs directly into the brain by overcoming $\mathrm{BBB}$ and enhancing the transportation of drug molecules through this barrier (as shown in Figure 1). These strategies are mainly divided into three categories according to their function and characteristic:-1) Invasive technique. 2) Non-invasive. 3) Recent techniques for BBB disruption.

\section{INVASIVE TECHNIQUE}

\section{Chemical disruption of blood brain barrier}

There are several invasive techniques which are commonly used to disrupt $\mathrm{BBB}$ and enhance the delivery of the drug into the brain. Osmotic disruption of $\mathrm{BBB}$ is one of the invasive technique in which temporary shrinkage of endothelial cells occurs which leads to the opening of tight junction and causing leakage of the drug to the CNS. ${ }^{9}$

According to mechanism $\mathrm{BBB}$ opening effects of bradykinin are due to activation of $\mathrm{B}_{2}$ receptor and leakage of endothelial cells which are 


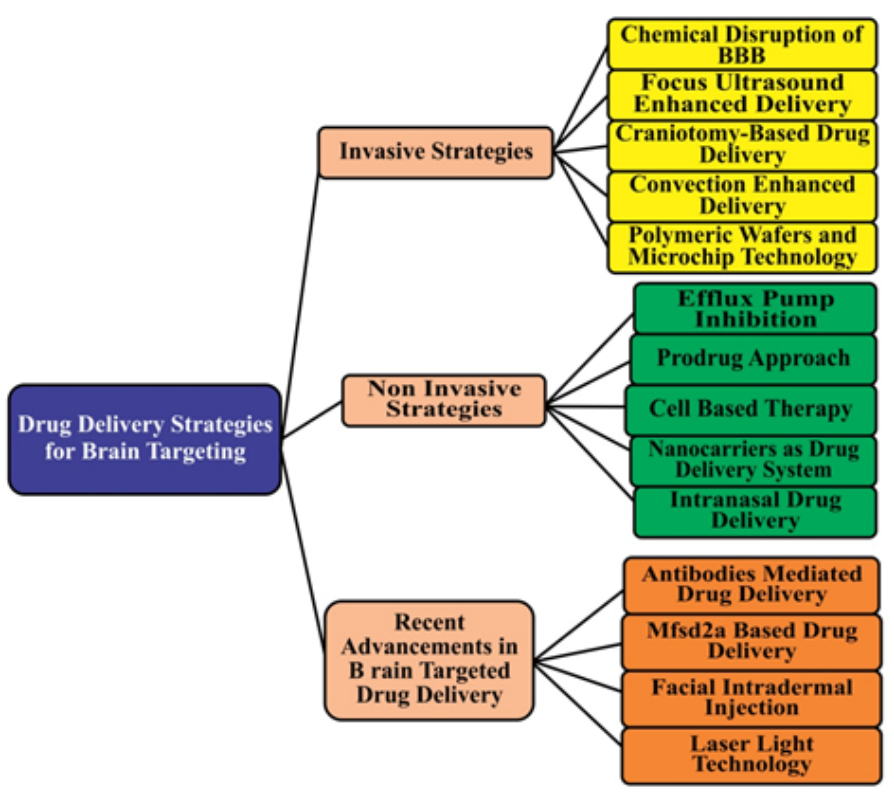

Figure 1: Depicting different strategy used in nasal drug delivery for brain targeting.

based on modulation of caveolin-1 and caveolin- $2^{10}$ the effectiveness of vasoactive agents could be maintained by conjugating into the surface of nanoparticles. Transplanting liposome with peptidase inhibitor bradykinin and etoposide promote the transportation of encapsulated drugs across BBB. ${ }^{11}$

\section{Focus ultrasound enhanced delivery}

The use of ultrasound waves for opening BBB reversibly and transiently is another versatile approach used for enhancing drug transportation to CNS. Ultrasound based drug delivery employs micro bubbles as a contrast agent. ${ }^{12}$ These bubbles worked on acoustic energy principles and administered systemically to apply pressure on endothelial cells and open the tight junction which increases the permeability of BBB and improved delivery of the drug to the brain. These micro bubbles are made of semi-rigid lipids and have a diameter of $1-10 \mu \mathrm{m}$. High intensity focused ultrasound strategy is applied for reversible disruption of BBB and advancement of drug distribution to the brain in a controlled way without any toxicity to brain parenchyma tissues. This strategy is mainly used for tumour-targeted delivery of drugs, genes and antibodies. ${ }^{13}$

\section{Craniotomy based drug delivery}

It is a direct way of targeting the specific part of the brain without any exposure to the peripheral organ. It is done via intracerebral or intraventricular injection. In the case of intraventricular delivery, a drug reservoir implanted subcutaneously in the scalp provided the controlled release of a drug and connected to the ventricles within the brain via an outlet catheter. ${ }^{14}$ This system directly delivers the drug to the ventricles and sub arachnoids part of the brain. In the case of the intracerebral system, it directly injects or infuses the drug into the brain parenchyma through the catheter and controlled devices regulate the delivery. It depends on the diffusion mechanism and provides a slow distribution of drugs in the brain. ${ }^{15}$

\section{Convection enhanced delivery}

It is used to overcome the disadvantage of the intracerebral delivery system. This system employs a continuous infusion method and pressure gradient to administered a large volume of drugs at target tissues via an intracranial catheter. CED has some limitation drug exposure to neighbouring tissue, the problem in designing the optimal formulations and sub-therapeutic level of drug in the targeted area. Combinations of CED with liposome enhance the efficiency of CED for brain tumor targeting. ${ }^{16}$

\section{Polymeric wafers and microchip technology}

Improvement in polymer technology led to the expansion of polymeric devices for controlled and targeted delivery of therapeutic moieties. Bypassing the $\mathrm{BBB}$ and achieving the control release delivery of drug at the intracranial tumour with the help of polymer device is a big accomplishment in the field of polymeric nanotechnology. Poly anhydride based wafers were inserted into the tumour resection area, crosses the BBB and gradually release the drug and distribute it into the brain and targeted site. Clinically polymeric wafers used local strategy for crossing $\mathrm{BBB}$ and providing the sustained release of antineoplastic drugs at the tumour-targeted site. Now According to clinical trials, local drug delivery based on polymeric anhydride polymer gave an efficacious result in animals without major organ toxicity. ${ }^{17}$

Programmed microchips knew as intracranial devices which are implanted to control the release of drug at the targeted site. There are two different types of chips - a) microelectromechanical system. b) Passive chip. Through these technologies single or multiple doses, delivery can be accomplished. Active microchips are based on microelectromechanical system which comprises a drug-filled reservoir on a silicon chip. It provides a high release of drug at the targeted site. ${ }^{18}$ Active chip technology allows the development of multiple reservoirs that contain separate drugs to be released at the time or at different time intervals. Passive chip release drug on moderate degradation of polymeric film enclosing reservoir. On-demand of the therapy, these chips also deliver multiple drugs. ${ }^{19}$

\section{NON-INVASIVE STRATEGY}

Non-invasive strategy utilizes the endogenous mechanism for the transport of the drug across BBB. It includes the prodrug approach, efflux pump inhibition and chemical modulation of $\mathrm{BBB}$, the alternative route of administration and nano carrier-based drug delivery to the brain.

\section{Prodrug approach}

In this approach by increasing the lipophilicity character of drug BBB permeation ability can be accelerated. Basically prodrug approach is a process of chemical modification of active pharmaceutical ingredients to modify the lipophilic behaviour and increasing the permeation ability with its water solubility. A targeted prodrug is a combination of chemical constituent and parent drug which is designed to offer an enzyme and transport system at the targeted site for converting it to an active moiety. This approach follows redox chemical delivery for saving the chemotherapeutic potential of mustard alkylating agents. As redox derivative of alkylating agents cross the BBB and stay in the brain for a longer time. ${ }^{20}$

\section{Efflux pump inhibition}

Another barrier for constructive drug delivery to the brain is due to the presence of the efflux pump in BBB. Efflux is due to the active pglycoprotein ( $\mathrm{p}-\mathrm{GP}$ ) present on the apical membrane of endothelial cells of $\mathrm{BBB}$ which results in poor drug accessibility at the targeted brain tissue. More likely p-GP has a close association with lipophilicity and cationic drugs. Drugs with low molecular weight are the substrate of $\mathrm{p}-\mathrm{GP}$ and are prohibited to enter the brain. So, inhibition of p-GP efflux is a helpful approach to retain the therapeutic efficacy of the potent drug. ${ }^{21}$ It was seen that the administration of first-generation p-GP efflux associated 
with cytochrome $\mathrm{p}_{4503 \mathrm{~A}}$ (CYP3A) enzyme inhibitor was toxic. While the second-generation strong $\mathrm{p}-\mathrm{GP}$ inhibitor showed less toxicity related to issues and the third generation inhibitor which has proved safety and not having any effect on CYP3A enzyme inhibitions. For avoiding the serious adverse effect which was associated with efflux inhibitor, dual therapy of efflux inhibitor is combined with nanoparticles was explored. Combinations of $\mathrm{p}$-GP and multidrug resistance-associated protein (MRP) families are located on the endothelial cell which is responsible for the efflux of the cationic molecule. As the prime function of $\mathrm{p}-\mathrm{GP}$ and MRP is to protect the brain from entering the harmful chemical substrate which inhibits the entry of drugs to the brain. ${ }^{22}$

\section{Cell-based therapy}

Cell-based therapy has retained most attention due to its capability of delivering a variety of drug effectiveness and treating a neurological disorder and brain tumours. In this therapy macrophages and different type of stem cells are used as a carrier for delivery of the drug to the brain. As macrophages can travel through the brain by paracellular and transcellular mechanisms. ${ }^{23}$ In the case of brain tumour and some inflammatory conditions, macrophages are engaged and infiltrated towards the brain. However, macrophages are an appropriate candidate for targeted drug delivery of nano particles with some of their special features like diagnosis and imaging of agents to the brain tumors and neurodegenerative diseases. Stem cells can be used as a vector for delivery of cytokines, oncolytic viruses and some suicides genes to the brain. ${ }^{24}$

\section{Nanocarrier as a drug delivery system}

In today's scenario nano carrier has got more attention and is the area of interest for researchers due to their capability as the permeation enhancement nano vehicle of a therapeutic molecule across BBB. Based on the study it was found that nanocarrier was having the capability of targeted site-specific delivery of different drug-like anticancer, antiAlzheimer, anti-Parkinson and protease inhibitor which made them more suitable candidate for the carrier of neurological disorder and brain tumor-targeted delivery. A combination of polymeric nanoparticles and $\mathrm{SLN}_{\mathrm{S}}$ are also effective nano vehicles for increasing the brain targeting efficiency of the drug. ${ }^{25}$

\section{Intranasal drug delivery}

It was found that drugs administered through the nasal route of administration were absorbed into the systemic circulation. While the drug absorption within nasal respiratory epithelium follows transcellular and paracellular absorption with carrier-mediated transport and absorption of the drug through transcytosis mechanism. ${ }^{26}$ However, it was a big challenge of delivering the drug through the nasal cavity to the brain due to the presence of $\mathrm{BBB}$ mediated restriction. But the administration of drug deep into the nasal cavity which approached nasal mucosa led to direct transportation of drug into the brain via specific olfactory pathways which consist of olfactory neuron carrying drug from olfactory mucosa to the brain which is a slow process of drug transportation. On the other hand, the olfactory epithelium pathway is a more confined and faster way of drug transportation. In this drug is directly passed through olfactory epithelium by paracellular mechanism into parenchymal space and is directly transferred into the brain. ${ }^{27}$

\section{RECENT ADVANCEMENT IN BRAIN TARGETED DRUG DELIVERY}

\section{Antibodies mediated drug delivery}

Antibodies based therapy become most prominent over the last decade. But the restriction created by $\mathrm{BBB}$ and low permeability of antibodies in the brain limit the potential of antibodies mediated therapy used for neurological diseases. However monoclonal antibodies (mAb) are much larger so they are not able to cross $\mathrm{BBB}$ as small molecules do and move towards the targeted site in the brain. Till the date, $\mathrm{mAb}$ has been approved for brain targeting strategies but several $\mathrm{mAb}$ are under clinical trials generally for the treatment of Alzheimer's diseases. ${ }^{28} \mathrm{~A}$ bispecific antibody (bsAb) is recently and newly developed $\mathrm{Ab}$ having two binding specificities. bsAb is incorporated for chemotherapy with a single binding specific target to the tumour cell and other targets the antigen present on the immune cell. The application of bsAb is targeted delivery across $\mathrm{BBB}$. Many of the bsAb which crosses were formulated and evolved for achieving brain targeted delivery like bsAb with transferrin receptor (TFR) binding domain for crossing BBB and single-chain variable region fragments specific against amyloid beta-peptide. ${ }^{29}$

Major Facilitator Super family Domain containing 2A (Mfsd2a) based drug delivery strategy. This is a novel approach for brain targeted drug delivery as Mfsd2a present over the surface of endothelial cells present in $\mathrm{BBB}$, which prohibit the transportation of molecule through transcytosis and facilitate the transport of specific lysophosphatidylcholine (LPC) derivative. Mfsd2a works on two principles one of which is inhibition of Mfsd2a which promotes transcytosis in endothelium and which results in the enhancement of $\mathrm{BBB}$ permeation. ${ }^{30}$ Whereas LPC acts as a carrier for small molecule and is transported by Mfsd2a across BBB. The second approach involves LPC mediated delivery which resembles parallelly to glucose transporter and L type amino acid transporter based transport. ${ }^{31}$

\section{Laser light-based technology}

This technology is more favourable for BBB disruption and can be helpful in glioma targeted delivery. As the laser light causes a defect in the membrane of endothelial cells due to which endothelial cells become leaky and allows the transportation of therapeutic agents to the parenchymal tissues. Later it was found that the combination therapy of laser aminolevulinic acid open the tight junction present between the endothelial cells for a longer period. However, the laser cannot be combined with other several strategies to penetrate BBB disruption. Ultra short laser pulse technique was adopted for the successful transportation of nanoparticle genetically engineered viruses and numerous therapeutic agents to the brain. ${ }^{32}$

\section{Facial intradermal injection}

Facial intradermal injection crosses the BBB through trigeminal neuronal connection. Trigeminal pathway contains vasculature; perineurium and epineurium interlink facial skin with the brain. The trigeminal pathway is used in facial intradermal targeting of the brain. ${ }^{33}$

\section{CONCLUSION}

According to literature it has been declared that brain targeting via nasal drug delivery is a fascinating approach. However, some of the alterations like surface modification of nanocarriers and the introduction of some specific ligand have provided some useful information and progress in the field. Modification of carriers by nano particles, micelles, liposome has led to a new era of drug delivery system commonly delivery of peptide and protein-based therapeutic agents to the brain. Nasal drug delivery serves some limitations like only specified and small quantity can be administered with its increasing molecular weight. Some effective non-invasive treatments of nasal delivery have some limitations because of the presence of dynamic barriers like BBB and CSF. However, there is a commanding challenge to increase the potency of effective drug targeting to the brain which can be achieved by a non-invasive and 
effective approach like intranasal drug delivery through which obstacle offered by BBB can be controlled.

\section{CONFLICT OF INTEREST}

The authors declare that there are no conflicts of interest.

\section{ABBREVIATIONS}

BBB: blood brain barrier; CNS: Central Nervous System; CSF: Cerebro Spinal Fluid; ND: Neurodegenerative Disease; SLN: Solid Lipid Nano Particles; TFR: Transferrin Receptor; mAb: Monoclonal Antibody; bsAb: Bispecific Antibody; CED: Convection Enhanced Delivery; p-GP: P Glycoprotein; CYP3A: Cytochrome $\mathrm{P}_{450} 3 \mathrm{~A}$; LPC: lysophosphatidylcholine; Mfsd2a: Major Facilitator Superfamily Domain containing $2 \mathrm{~A}$.

\section{REFERENCES}

1. Prince M, Wimo A, Guerchet M, Ali GC, Wu YT, Prina M. World Alzheimer Report: The Global Impact of Dementia, An analysis of prevalence, incidence, cost and trends. Alzheimer's Dis Int. 2015;84:01-92.

2. Nigar S, Pottoo FH, Tabassum N, Verma SK, Javed MN. Molecular Insights into the Role of Inflammation and Oxidative Stress in Epilepsy. JOAMPS. 2016;10:19.

3. Rao AT, Degnan AJ, Levy LM. Genetics of Alzheimer disease. Am J Neuroradiol. 2014;35(3):457-8.

4. Javed MN, Alam MS, Pottoo FH. Metallic nanoparticle alone and/or in combination as novel agent for the treatment of uncontrolled electric conductance related disorders and/or seizure, epilepsy and convulsions. PCT Pat Appl. 2017. WO2017060916 A1 2017.

5. Haque S, Shadab M, Fazil M, Kumar M, Sahni JK, Ali J, et al. Venlafaxine loaded chitosan NPs for brain targeting, Pharmacokinetic and pharmacodynamic evaluation. Carbohydr Polym. 2012;89(1):72-9.

6. Hanson LR, Frey WH. Intranasal delivery by passes the blood brain barrier to Target therapeutic agents to the central nervous system and treat neurodegenerative disease. BMC Neurosci. 2008;9(S3): S5.

7. Johnson $\mathrm{PH}$, Quay SC. Advances in nasal drug delivery through tight junction Technology. Expert Opin Drug Deliv. 2005; 2(2):281-98.

8. Chein YW, Su KS, Chang SF. Intranasal drug delivery for systemic medications. Crit Rev Ther Drug Carrier Syst. 1987;4(2):67-194

9. Rapoport SI, Robinson PJ. Tight-junctional modification as the basis of osmotic opening of the blood-brain barrier. Ann NY Acad Sci. 1986;481(1):250-67.

10. Liu LB, Xue YX, Liu YH. Bradykinin increases the permeability of the blood tumor barrier by the caveolae-mediated transcellular pathway. J Neuro-Oncol. 2010;99(2):187-94

11. Rainov NG, Ikeda K, Qureshi NH, Grover S, Herrlinger U, Pechan $P$, et al. Intraarterial delivery of adenovirus vectors and liposome-DNA complexes to experimental brain neoplasms. Hum Gene Ther. 1999;10(2):311-8.

12. Horodyckid C, Canney M, Vignot A, Boisgard R, Drier A, Huberfeld G, et al. Safe long-term repeated disruption of the blood-brain barrier using an implantable ultrasound device: A multiparametric study in primates. J Neurosurg. 2017;126(4):1351-61.

13. Fry WJ, JrMosberg WH, Barnard JW, Fry FJ. Production of focal destructive lesions in the central nervous system with ultrasound. J Neurosurg. 1954; $11(5): 471-8$.

14. Begley DJ, Squires LK, Zlokovic BV, Mitrovic DM, Hughes CCW, Revest PA, et al. Physiology and pharmacology of blood brain barrier. J Neurochem. 1998;55(4):1222-30

15. Nicholson C, Sykova E. Extracellular space structure revealed by diffusion analysis. Trends Neuro Sci. 1998;21(5):207-15.

16. Fiandaca MS, Forsayeth JR, Forsayeth PJ, Bankiewicz KS. Image-guided convection-enhanced delivery platform in the treatment of neurological diseases. Neurotherapeutics. 2008;5(1):123-7.

17. Kawano K, Watanabe M, Yamamoto T, Yokoyama M, Opanasopit P, Okano T, et al. Enhanced antitumor effect of camptothecin loaded in long-circulating polymeric micelles. J Control Release. 2006;112(3):329-32.

18. Staples M. Microchips and controlled-release drug reservoirs. WIREs Nanomed Nanobiotech. 2010;2:400-17.

19. Sutradhar KB, Sumi CD. Implantable microchip: The futuristic controlled drug delivery system. Drug Deliv. 2016;23(1):1-11.

20. Singh RK, Prasad DN, BhardwajTN. Design, synthesis, chemical and biological evaluation of brain targeted alkylating agent using reversible redox prodrug approach. Arab J Chem. 2017;10(3):420-9.

21. Minocha M, Khurana V, Qin B, Pal D, Mitra AK. Enhanced brain accumulation of pazopanib by modulating P-gp and Bcrp1 mediated efflux with canertinib or erlotinib. Int J Pharm. 2012;436(1-2):127-34.

22. Shukla S, Ohnuma S, Ambudkar SV. Improving cancer chemotherapy with modulators of ABC drug transporters. Curr Drug Targets. 2011;12(5):621-30.

23. Engelhardt B, Ransohoff RM. Capture, crawl, cross: The T cell code to breach the Blood brain barriers. Trends Immunol. 2012;33(12):579-89.

24. Hong X, Miller C, Savant-Bhonsale S, Kalkanis SN. Antitumor treatment using interleukin-12- secreting marrow stromal cells in an invasive glioma model. Neurosurgery. 2009;64(6):1139-46 (discussion 1146-1137).

25. Bruun J, Larsen TB, Jølck RI, Eliasen R, Holm R, Gjetting T, et al. Investigation of enzyme-sensitive lipid nanoparticles for delivery of siRNA to blood-brain barrier and glioma cells. Int J Nanomedicine. 2015;10:5995-6008.

26. Roy S. Strategic drug delivery targeted to the brain: A review. Der Pharmac Sin 2012;3(1):76-92

27. Sanjay D, Mahantil B, Majumder B. Nasal drug delivery: An approach of drug delivery through nasal route. Der Pharmac Sin. 2011;2(3):94-106.

28. Alzforum AD. http://www.alzforum.org/news/conferencecoverage/ aducanumab-solanezumab-gantenerumab-data-liftcrenezumab-well. 2015.

29. Tengfei L, Vandesquill M, Koukouli F, Dudeffant C, Youssef I, Lenormand P, et al. Camelid single-domain antibodies: a versatile tool for in vivo imaging of extracellular and intracellular brain targets. J Control Release. 2016;243:1-10.

30. Gu C, Ben-Zvi A. Modulating or treating permeability of blood-brain barrier by administering inhibitor of gene, or agonist of gene, or gene expression product e.g. major facilitator superfamily domain-containing protein 2(Mfsd2A) to human, Harvard College (Hard-C), International application number: PCT/ US2014/043395, International publication number: WO 2014/205338 A2. 2016.

31. Pardridge WM. Blood-brain barrier endogenous transporters astherapeutic targets: A new model for small molecule CNS drug discovery. Expert Opin Ther Targets. 2015;19(8):1059-72.

32. Choi M, Ku T, Chong K, Yoon J, Choi C. Minimally invasive molecular delivery into the brain using optical modulation of vascular permeability. Proc Natl Acad Sci USA. 2011;108(22): 9256-61.

33. Yu XC, Yang JJ, Jin BH, Xu HL, Zhang HY, Xiao J, et al. A strategy for bypassing the blood-brain barrier: facial intradermal brain targeted delivery via the trigeminal nerve. J Control Release. 2017;258:22-33.

Article History: Submission Date : 28-05-2020; Revised Date : 11-06-2020; Acceptance Date : 21-07-2020.

Cite this article: Kushwaha SKS, Kushwaha N, Fatma B, Pandey P. Nanostructured Lipid Carriers (NLC): A Targeting Approach to the Brain via Intranasal Administration. Int. J. Pharm. Investigation, 2020;10(3):253-6 